University of New Orleans

ScholarWorks@UNO

$1-1-2002$

\title{
Symmetrically coated pellicle beam splitters for dual quarter-wave retardation in reflection and transmission
}

\author{
Rasheed M.A. Azzam \\ University of New Orleans, razzam@uno.edu \\ Fadi A. Mahmoud
}

Follow this and additional works at: https://scholarworks.uno.edu/ee_facpubs

Part of the Electrical and Electronics Commons

\section{Recommended Citation}

Rasheed M. A. Azzam and Fadi A. Mahmoud, "Symmetrically Coated Pellicle Beam Splitters for Dual Quarter-Wave Retardation in Reflection and Transmission," Appl. Opt. 41, 235-238 (2002)

This Article is brought to you for free and open access by the Department of Electrical Engineering at ScholarWorks@UNO. It has been accepted for inclusion in Electrical Engineering Faculty Publications by an authorized administrator of ScholarWorks@UNO. For more information, please contact scholarworks@uno.edu. 


\title{
Symmetrically coated pellicle beam splitters for dual quarter-wave retardation in reflection and transmission
}

\author{
Rasheed M. A. Azzam and Fadi A. Mahmoud
}

\begin{abstract}
A trilayer pellicle that consists of a high-index center layer that is symmetrically coated on both sides by a low-index film can be designed to produce differential reflection and transmission phase shifts of $\pm 90^{\circ}$ at oblique incidence and equal throughput for the $p$ and the $s$ polarizations. Such a device splits a beam of incident linearly polarized light into two orthogonal circularly polarized components that travel in well-separated angular directions. Examples of infrared dual quarter-wave retarders that use a symmetrically coated Ge pellicle at $77^{\circ}$ angle of incidence are presented. A 50-50\% splitter requires a symmetric pellicle with at least five layers. Error analysis shows that the thicknesses of the high-index layers must be tightly controlled. These circular polarization beam splitters are intended for operation with a well-collimated light source and can be used as the basis of a novel circular polarization Michelson interferometer. (C) 2002 Optical Society of America

OCIS codes: $120.2130,260.5430,230.5440$.
\end{abstract}

\section{Introduction}

Quarter-wave retarders are widely used in optics for polarization analysis and control and as linear-tocircular or circular-to-linear polarization transformers. ${ }^{1}$ They are usually designed by use of natural or induced linear birefringence, total internal reflection, or interference in thin films.

Coherent multiple-beam interference in a uniform, tilted, thin dielectric slab (pellicle) produces a differential transmission phase shift between the $p$ and $s$ linear polarizations (parallel and perpendicular to the plane of incidence, respectively) or a net retardance $\Delta_{t}<90^{\circ}$. Therefore a tilted pellicle of a homogeneous optically isotropic material acts as the simplest possible fractional-wave (less than quarterwave) retardation plate. ${ }^{2}$ Light interference in a suitably designed tilted bilayer membrane realizes a quarter-wave retardation (QWR) in transmission $\left(\Delta_{t}=90^{\circ}\right)$ at a high incidence angle. ${ }^{3}$ In general, these pellicle retarders have unequal throughput for

R. M. A. Azzam (razzam@uno.edu) is with the University of New Orleans, Department of Electrical Engineering, Lakefront, New Orleans, Louisiana 70148. F. A. Mahmoud is with Adaptec, Incorporated, 691 South Milpitas Boulevard, Milpitas, California 95035.

Received 21 May 2001.

0003-6935/02/010235-04\$15.00/0

(C) 2002 Optical Society of America the $p$ and $s$ polarizations, hence they exhibit diattenuation. ${ }^{4}$

In this paper we show that dual QWR in transmission and reflection $\left(\Delta_{t}=+90^{\circ}\right.$ and $\left.\Delta_{r}=-90^{\circ}\right)$ can be produced, without diattenuation, by use of a trilayer pellicle that consists of a high-index center layer that is symmetrically coated on both sides with a lowindex thin film. Therefore a simple circular polarization beam splitter is realized, for the first time to our knowledge, by use of conventional (nongyrotropic) thin-film optics. Earlier circular polarization beam splitters are based on reflection by chiral media $^{5}$ or diffraction by liquid-crystal gratings. ${ }^{6}$

\section{Design Procedure}

Changes in the state of polarization of light upon transmission (reflection) by a trilayer pellicle, Fig. 1, are determined by the ratios of complex-amplitude transmission (reflection) coefficients for the $p$ and the $s$ polarizations:

$$
\begin{gathered}
\tau=T_{p} / T_{s}=\tan \Psi_{t} \exp \left(j \Delta_{t}\right), \\
\rho=R_{p} / R_{s}=\tan \Psi_{r} \exp \left(j \Delta_{r}\right) .
\end{gathered}
$$

The transmission and reflection coefficients of a multilayer system are determined by use of the scattering matrix method. ${ }^{7}$ For a monochromatic light beam of a given wavelength $\lambda$, which is incident on 


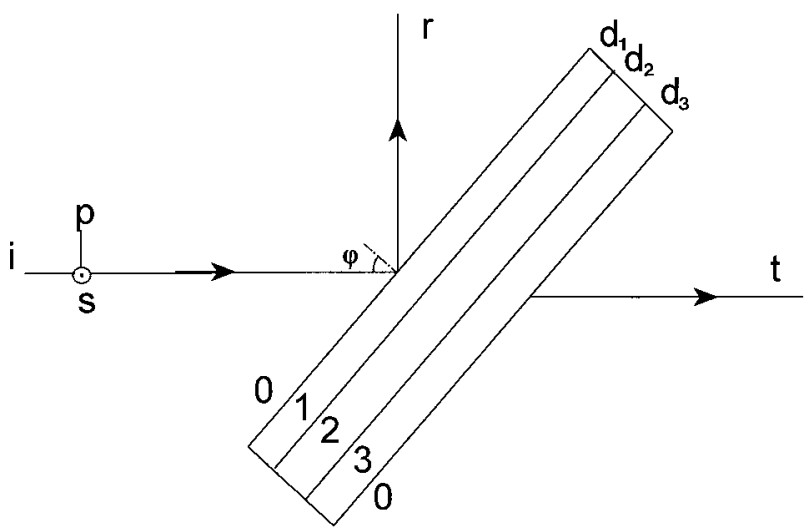

Fig. 1. Reflection and transmission of light by a tilted pellicle that consists of three optically isotropic layers (1, 2, and 3 ) with uniform thicknesses $d_{1}, d_{2}$, and $d_{3}$. The pellicle is immersed in a transparent ambient medium (0). The linear polarizations $p$ and $s$ are parallel and perpendicular to the plane of incidence, respectively, and $\varphi$ is the angle of incidence.

the pellicle at an angle of incidence $\varphi$, the ellipsometric functions ${ }^{7}$ of Eqs. (1) and (2) can be put in the form

$$
\begin{aligned}
& \tau=f\left(\varphi, n_{1}, n_{2}, \zeta_{1}, \zeta_{2}\right), \\
& \rho=g\left(\varphi, n_{1}, n_{2}, \zeta_{1}, \zeta_{2}\right) .
\end{aligned}
$$

We assume a symmetrically coated pellicle, Fig. 1, with identical outer layers 1 and 3 of the same (low) refractive index $\left(n_{3}=n_{1}\right)$ and the same normalized thickness $\left(\zeta_{3}=\zeta_{1}\right)$ and a center layer 2 with a different (high) refractive index $n_{2}$ and normalized thickness $\zeta_{2}$. The immersion medium 0 on both sides of the pellicle is assumed to be air or vacuum with refractive index 1. All media are assumed to be homogeneous, optically isotropic, and separated by parallel-plane boundaries. The normalized thickness $\zeta_{i}$ of the $i$ th film is defined by

$$
\zeta_{i}=d_{i} / D_{i}, i=1,2,3,
$$

where

$$
D_{i}=(\lambda / 2)\left(n_{i}{ }^{2}-\sin ^{2} \varphi\right)^{-1 / 2}, \quad i=1,2,3
$$

is the angle-of-incidence-dependent film thickness period. To achieve pure QWR in transmission, the following two conditions must be satisfied simultaneously:

$$
\begin{aligned}
|\tau| & =\left|T_{p}\right| /\left|T_{s}\right|=1, \\
\arg (\tau) & =\Delta_{t}= \pm 90^{\circ} .
\end{aligned}
$$

For an all-transparent pellicle,

$$
|\rho|=\left|R_{p}\right| /\left|R_{s}\right|=1
$$

is satisfied simultaneously with Eq. (7). Equations (7) and (9) indicate the absence of diattenuation in transmission and reflection. Finally, to obtain QWR in reflection, we should also have

$$
\arg (\rho)=\Delta_{r}= \pm 90^{\circ} .
$$

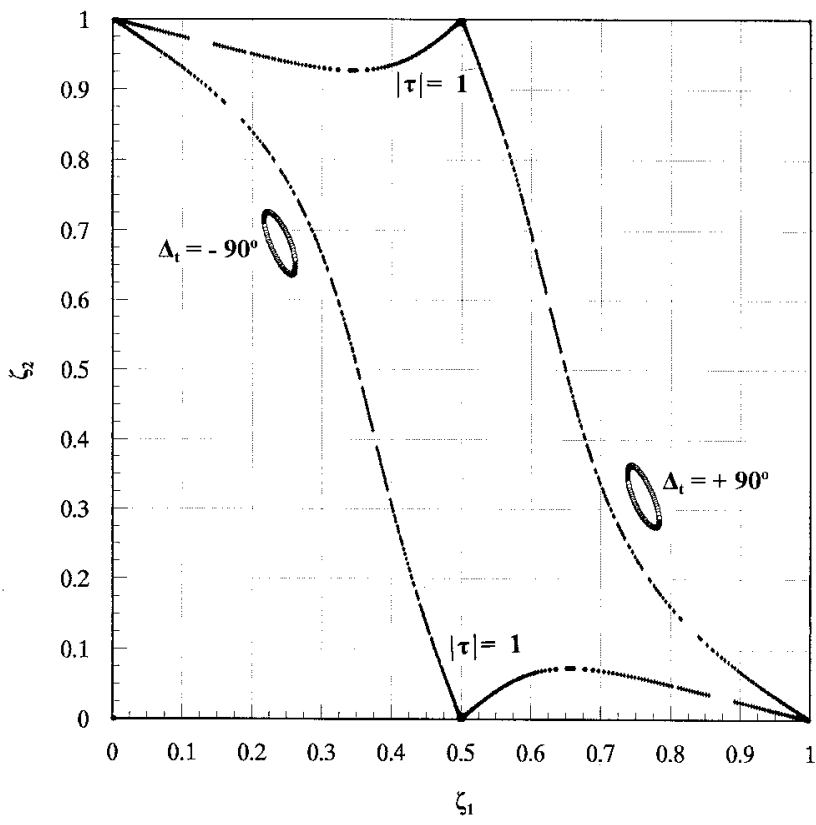

Fig. 2. Loci of multiple solutions $\left(\zeta_{1}, \zeta_{2}\right)$ of Eq. (8) for symmetric trilayer transmission quarter-wave retarders for both $\Delta_{t}=+90^{\circ}$ and $\Delta_{t}=-90^{\circ}$ are presented by the closed contours. Superimposed are the corresponding solution loci for Eq. (7), $|\tau|=1$, for a coated Ge trilayer (with indices $1.35,4,1.35$ ) at $\varphi=75^{\circ}$ angle of incidence. Note that the two solution loci do not intersect.

For given film refractive indices $\left(n_{1}, n_{2}\right)$ and a given angle of incidence $\varphi$, the normalized film thicknesses $\left(\zeta_{1}, \zeta_{2}\right)$ are iterated on to satisfy Eqs. (7)-(10) individually or simultaneously. To achieve QWR at the lowest possible angle of incidence, by use of a symmetric trilayer pellicle in air, the refractive-index contrast between the center and the outer layers should be sufficiently high. In Section 3 we consider a pellicle that consists of a high-index center layer of Ge $\left(n_{2}=4\right)$ that is symmetrically coated by a lowindex (fluoride ${ }^{8}$ ) thin film $\left(n_{1}=1.35\right)$ for incident infrared radiation.

\section{Symmetric Trilayer Pellicle as Dual Transmission and Reflection Quarter-Wave Retardation at $75^{\circ}$ Angle of Incidence}

As a specific example, we take $\left(n_{1}, n_{2}, n_{3}\right)=(1.35,4$, 1.35), which corresponds to a symmetrically coated pellicle, with a Ge center layer and fluoride outer coating at 10.6- $\mu \mathrm{m}\left(\mathrm{CO}_{2}\right.$-laser $)$ wavelength and $\varphi=$ $75^{\circ}$ angle of incidence. This operating angle is $7^{\circ}$ lower than that at which the bilayer membrane of Ref. 2 functions as a QWR and leads to an angular separation of $30^{\circ}$ between the reflected and the transmitted beams.

Figure 2 shows the loci of multiple solutions $\left(\zeta_{1}, \zeta_{2}\right)$ of Eq. (8) for transmission quarter-wave retarders for both $\Delta_{t}=+90^{\circ}$ and $\Delta_{t}=-90^{\circ}$ as represented by the two small closed contours.

Superimposed on Fig. 2 are the corresponding solution loci of Eq. (7), $|\tau|=1$, for the same trilayer. The two solution loci do not intersect, hence Eqs. (7) 
and (8) cannot be satisfied simultaneously. Consequently, it is not possible to achieve QWR without diattenuation at $\varphi=75^{\circ}$. The azimuth of incident linearly polarized light can be adjusted to circularly polarize one of the two split beams at a time, but not both at the same time.

To quote a specific design, a trilayer with $\left(\zeta_{1}, \zeta_{2}\right)=$ $(0.2430,0.7066)$ and least metric thicknesses $\left(d_{1}, d_{2}\right)$ $=(1365.5,964.7) \mathrm{nm}$ functions as QWR in both reflection and transmission (with opposite signs of retardance) at $\varphi=75^{\circ}$. The intensity transmittances for the $p$ and $s$ polarizations are $39.13 \%$ and $18.01 \%$, respectively. Incident linearly polarized light with an azimuth of $55.84^{\circ}$, measured from the $p$ direction, produces a circularly polarized transmitted beam.

In general, we find that, for symmetric pellicles, if QWR is achieved in transmission, QWR is also obtained simultaneously in reflection, with $\pm 90^{\circ}$ retardance in the two split beams. However, the reverse of this statement is not true, i.e., QWR in reflection does not guarantee QWR in transmission. This important conclusion (for which an analytical proof may be possible) extends to symmetric multilayer pellicles with any odd number of layers.

To satisfy Eqs. (7) and (8) simultaneously, and to achieve orthogonal circular polarizations in the two split beams at the same time, the angle of incidence $\varphi$ must be increased to just above $76^{\circ}$. In Section 4 we present a design that operates at $\varphi=77^{\circ}$.

\section{Symmetric Trilayer Pellicle as Dual Quarter-Wave Retardation without Diattenuation at $77^{\circ}$ Angle of Incidence}

We assume the same material system that we considered in Section 3. The solution loci of Eqs. (7) and (8) for the normalized film thickness $\left(\zeta_{1}, \zeta_{2}\right)$ appear in Fig. 3 at $\varphi=77^{\circ}$. Figure 4 shows the corresponding solution loci for Eqs. (9) and (10).

Figures 3 and 4 confirm that Eqs. (7) and (9) have the same solution, as one expects for a transparent system. Figure 4 shows that the solution loci for Eq. (10) consist of two closed contours that exactly coincide with the corresponding closed contours in Fig. 3, except that when $\Delta_{t}=+90^{\circ}, \Delta_{r}=-90^{\circ}$, and vice versa. Figure 4 indicates two additional solution branches for Eq. (10) for which QWR is achieved only in reflection but not in transmission.

The intersection points $x, y, u$, and $v$ in Figs. 3 and 4 represent solutions for which QWR is achieved simultaneously in transmission and reflection without diattenuation.

We further consider the design marked $x$ in Figs. 3 and 4. The normalized and least metric film thicknesses for this symmetric trilayer are $(0.225855$, $0.803674)$ and $(1281.0,1097.9) \mathrm{nm}$, respectively. The transmission and reflection differential phase shifts are $\Delta_{t}=-90^{\circ}$ and $\Delta_{r}=+90^{\circ}$, respectively. The polarization-independent (same for $p$ and $s$ ) transmittance and reflectance are $37.78 \%$ and $62.22 \%$, respectively. It is not possible to achieve dual QWR with equal split fractions by use of a trilayer pellicle in air. In Section 5 we briefly dis-

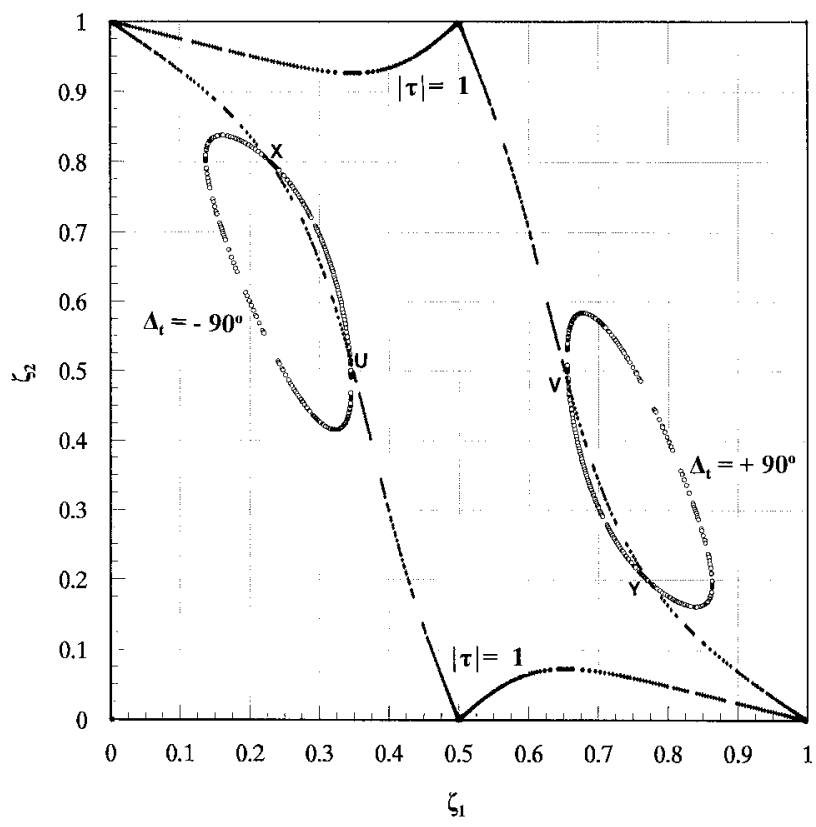

Fig. 3. Loci of multiple solutions $\left(\zeta_{1}, \zeta_{2}\right)$ of Eq. (8) for symmetric trilayer transmission quarter-wave retarders for both $\Delta_{t}=+90^{\circ}$ and $\Delta_{t}=-90^{\circ}$ are presented by the closed contours. Superimposed are the corresponding solution loci for Eq. (7), $|\tau|=1$, for a coated Ge trilayer (with indices $1.35,4,1.35$ ) at $\varphi=77^{\circ}$ angle of incidence. The intersection points $x, y, u$, and $v$ represent trilayer pellicles that function as dual QWR in transmission and reflection without diattenuation.

cuss a 50-50\% design using a symmetric five-layer pellicle.

It is important to specify the sensitivity of this design to small deviations of incidence angle, wavelength, and film thicknesses from their design values. To maintain the phase error in reflection and transmission to within $\pm 1^{\circ}, \varphi$ and $\lambda$ must be kept to within $\pm 0.2^{\circ}$ and $\pm 40 \mathrm{~nm}$ of their design values, respectively. This indicates that this circular polarization beam splitter is suited mainly for well-collimated laser radiation. The associated tolerances for the thicknesses of layers 1,2 , and 3 , for the same $\pm 1^{\circ}$ phase error, are given by $\pm 4 \%, 0.5 \%$, and $4 \%$, respectively. Thus stringent thickness control is required mainly for the middle high-index layer.

\section{Symmetric, Five-Layer, $50-50 \%$ Pellicle Beam Splitter with Dual Quarter-Wave Retardation without Diattenuation at $78^{\circ}$ Angle of Incidence}

Assume that we wish to design a circular polarization pellicle beam splitter (immersed in air) that satisfies all the following requirements simultaneously:

1. dual quarter-wave retardations with opposite signs in reflection and transmission,

2 . equal throughput for the $p$ and the $s$ polarizations (i.e., no diattenuation), and

3. $50-50 \%$ split ratio. 


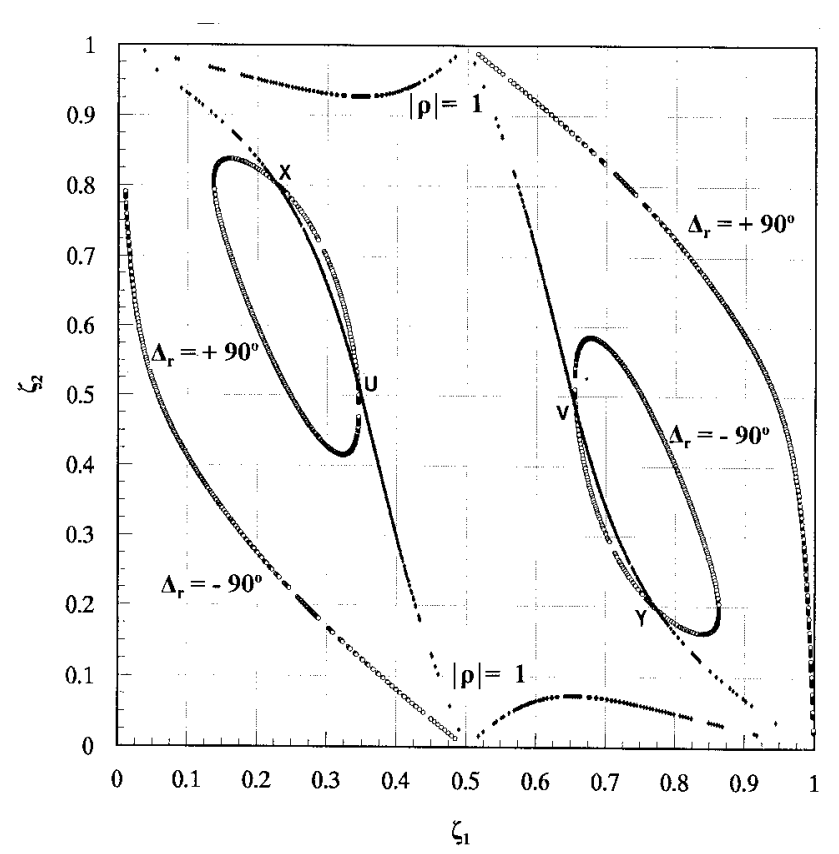

Fig. 4. Loci of multiple solutions $\left(\zeta_{1}, \zeta_{2}\right)$ of Eq. (10) for symmetric trilayer reflection quarter-wave retarders for both $\Delta_{r}=+90^{\circ}$ and $\Delta_{r}=-90^{\circ}$. There are four solution branches; the two branches represented by the closed contours coincide with the closed contours for transmission QWR in Fig. 3. Superimposed are the corresponding solution loci for Eq. (9), $|\rho|=1$, for the same coated Ge trilayer (with indices $1.35,4,1.35$ ) at $\varphi=77^{\circ}$ angle of incidence. The intersection points $x, y, u$, and $v$ represent trilayer pellicles that function as dual QWR in transmission and reflection without diattenuation.

To satisfy all these conditions by numerical experimentation, we find that a symmetric pellicle with at least five layers is required. The parameters of one such design are as follows:

- incidence angle $\varphi=78^{\circ}$, wavelength $\lambda=10.6$ $\mu \mathrm{m}$;

- film refractive indices $n_{1}=n_{3}=n_{5}=1.35$ and $n_{2}=n_{4}=4$

- normalized film thicknesses $\zeta_{1}=\zeta_{5}=0.022556$, $\zeta_{3}=0.36082$, and $\zeta_{2}=\zeta_{4}=0.932137$;

- metric film thicknesses $d_{1}=d_{5}=128.5 \mathrm{~nm}$, $d_{3}=2055.3 \mathrm{~nm}$, and $d_{2}=d_{4}=1273.7 \mathrm{~nm}$;

- differential reflection and transmission phase shifts $\Delta_{t}=-90.0002^{\circ}$ and $\Delta_{r}=+89.9998^{\circ}$;

- amplitude transmittance and reflectance ratios $|\tau|=0.999998$ and $|\rho|=1.000002$; and

- intensity reflectance and transmittances of $50.24 \%$ and $49.76 \%$, respectively.
Of the three conditions cited above, the first two are almost exactly satisfied, and the third is met to within $0.24 \%$. However, the main problem with this design is that it is sensitive to small thickness errors of the even-numbered (high-index) layers. For example, a $1 \%$ error of $d_{2}$ causes a phase error of approximately $10^{\circ}$. This makes this design impractical. To overcome this remaining problem, it appears that the multilayer pellicle design would probably have to be abandoned.

\section{Conclusion}

Pellicles are traditionally used as light, yet quite sturdy, beam splitters. ${ }^{9}$ In this paper we have shown that, by appropriate design, a trilayer pellicle that consists of a high-index $(\mathrm{Ge})$ center layer that is symmetrically coated with a low-index (fluoride) thin film can function as dual QWR in reflection and transmission simultaneously without introducing any diattenuation. To achieve a 50-50\% split ratio, a symmetric alternating (high-index and low-index) stack of at least five layers is required. Such a device functions as a circular polarization beam splitter for incident linearly polarized light, which can be used as the key element of a circular polarization Michelson interferometer. ${ }^{10}$

\section{References}

1. See, for example, W. A. Schucliff, Polarized Light (Harvard University, Cambridge, Mass., 1962).

2. D. A. Holmes, "Wave optics theory of rotatory compensators," J. Opt. Soc. Am. 54, 1340-1347 (1964).

3. R. M. A. Azzam and F. A. Mahmoud, "Tilted bilayer membranes as simple transmission quarter-wave plates," J. Opt. Soc. Am. A 18, 421-425 (2001).

4. R. A. Chipman, "Polarization analysis of optical systems," Opt. Eng. 28, 90-99 (1989).

5. S. D. Jacobs, K. A. Cerqua, K. L. Marshall, A. Schmid, M. J. Guardalben, and K. J. Skerrett, "Liquid-crystal laser optics: design, fabrication, and performance,” J. Opt. Soc. Am. B 5, 1962-1975 (1988).

6. J. A. Davis, J. Adachi, C. R. Fernandez-Pousa, and I. Moreno, "Polarization beam splitters using diffraction gratings," Opt. Lett. 26, 587-589 (2001).

7. R. M. A. Azzam and N. M. Bashara, Ellipsometry and Polarized Light (North-Holland, Amsterdam, 1987), Chap. 4.

8. E. Ritter, "Optical film materials and their applications," Appl. Opt. 15, 2318-2327 (1976).

9. J. A. Dobrowolski, "Optical properties of films and coatings," Handbook of Optics, M. Bass, ed. (McGraw-Hill, New York, 1995), Vol. 1, Chap. 42.

10. R. M. A. Azzam and F. A. Mahmoud, "Circular polarization Michelson interferometer," presented at the 1999 Annual Meeting of the Optical Society of America, Santa Clara, Calif., 26-30 Sept. 1999, paper TuXX66. 